\title{
Frontières
}

\section{La place de la mort dans l'Islam}

\section{Sarah Burkhalter}

Volume 13, numéro 1, automne 2000

URI : https://id.erudit.org/iderudit/1074255ar

DOI : https://doi.org/10.7202/1074255ar

Aller au sommaire du numéro

\section{Éditeur(s)}

Université du Québec à Montréal

ISSN

1180-3479 (imprimé)

1916-0976 (numérique)

Découvrir la revue

\section{Citer cet article}

Burkhalter, S. (2000). La place de la mort dans l'Islam. Frontières, 13(1), 73-80. https://doi.org/10.7202/1074255ar

\section{Résumé de l'article}

La vie après la mort tient une place centrale dans la religion islamique. L'affirmation de l'existence du Jour du Jugement et de la Résurrection est omniprésente dans le Coran. Elle représente en quelque sorte le point nodal du message prophétique. En effet, tout tend vers ce moment crucial du Jugement, qui donne sens à tous les gestes et toutes les pensées que le croyant a pu avoir de son vivant. En ce qui concerne les rites funéraires, il est intéressant de constater une certaine souplesse dans leur application. Seuls quelques-uns sont obligatoires, tous les autres ne sont que conseillés. De même, on remarque une variété des pratiques d'un bout à l'autre du monde musulman, et une liberté dans l'interprétation des textes. Ainsi, le degré d'obligation normative d'un rite ne correspond pas forcément avec son degré d'application par la population.
Ce document est protégé par la loi sur le droit d'auteur. L'utilisation des services d'Érudit (y compris la reproduction) est assujettie à sa politique d'utilisation que vous pouvez consulter en ligne.

https://apropos.erudit.org/fr/usagers/politique-dutilisation/ 


\section{Résumé}

La vie après la mort tient une place centrale dans la religion islamique. L'affirmation de l'existence du Jour du Jugement et de la Résurrection est omniprésente dans le Coran. Elle représente en quelque sorte le point nodal du message prophétique. En effet, tout tend vers ce moment crucial du Jugement, qui donne sens à tous les gestes et toutes les pensées que le croyant a pu avoir de son vivant. En ce qui concerne les rites funéraires, il est intéressant de constater une certaine souplesse dans leur application. Seuls quelques-uns sont obligatoires, tous les autres ne sont que conseillés. De même, on remarque une variété des pratiques d'un bout à l'autre du monde musulman, et une liberté dans l'interprétation des textes. Ainsi, le degré d'obligation normative d'un rite ne correspond pas forcément avec son degré d'application par la population.

Mots clés: Islam - mort - rites funéraires

\section{Abstract}

Life after death occupies a central place in the Islamic Religion. Affirmation of the existence of Judgment and Resurrection Day is omnipresent in the Koran. It represents, as it were, the nodal point of the prophetic message. Everything leans toward this crucial moment of Judgement, which imparts meaning to all deeds and all thoughts that the follower may have during his lifetime. As for funeral rites, it is interesting to note a certain flexibility in their application. Only a few are mandatory; all the rest are merely suggested. Likewise, one can find a variety of practices from one end of the Muslim World to the another as well as a freedom in how writings are interpreted. Thus, the extent of normative obligation of a rite does not necessarily correspond with the degree of how people apply it.

Key words: Islam - death-funeral rites

$\begin{array}{lllllllll}H & O & R & S & T & H & \text { E } & M & E\end{array}$

\section{La place de la mort dans I'Islam}

\section{Ô MON ENFANT, FÛT-CE LE POIDS D'UN GRAIN DE MOUTARDE, AU FOND D'UN ROCHER, OU DANS LES CIEUX OU DANS LA TERRE, ALLAH LE FERA VENIR (AU JOUR DU JUGEMENT DERNIER).}

- COR. $31 ; 16$

\begin{abstract}
Sarah Burkhalter,
licenciée en Sociologie et Histoire et Sciences des religions, a publié un mémoire de licence sur la question des cimetières en Suisse.
\end{abstract}

Il suffit d'ouvrir le Coran pour se rendre compte que la prédication du prophète Mohammed est centrée sur l'annonce de la fin des temps, de la Résurrection et du Jugement dernier. Déjà dans la sourate liminaire (fatiha), la plus récitée par les croyants, Allah n'est pas seulement appelé "Seigneur de l'univers", mais également "Maître du Jour de la rétribution ". L'affirmation de l'existence du Jour du Jugement et de la Résurrection est omniprésente et figure dans la plupart des sourates coraniques. Elle représente en quelque sorte le point nodal du message prophétique. C'est en effet l'existence de cet au-delà qui va justifier l'importance de la foi et l'accomplissement des rites. Les délices du Paradis et les affres de l'Enfer font l'objet de multiples descriptions, destinées à convaincre le destinataire de suivre la voie droite. Le fait de centrer son message sur l'annonce du Jour du Jugement ne constitue pas une particularité de
l'Islam. Tous les principaux prophètes de l'Ancien Testament l'ont fait. Mais alors que les livres prophétiques ne représentent qu'une partie de la Bible, le Coran est parsemé en son entier de ces annonces menaçantes du Jugement. Pourquoi une telle dramatisation de l'au-delà?

\section{DU CÔTÉ DE LA FOI}

Nous examinerons séparément les éléments de foi d'une part, de rites d'autre part. Par le fait que la croyance et le rite ne correspondent pas forcément l'un à l'autre, et qu'un rite ne répond pas automatiquement à une croyance, ni non plus que chaque élément de croyance débouche sur un rite précis, il est intéressant de les mettre en miroir l'un de l'autre. Nous précisons que nous donnons ici la conception généralement admise dans l'Islam, et que nous n'entrerons pas dans les détails distinguant les quatre écoles juridiques du sunnisme (chafiite, hanbalite, hanafite et malikite) ou l'islam shî'ite' . Nous nous basons uniquement sur le Coran et les Hadiths (paroles rapportées du Prophète), et non sur l'ensemble des développements juridiques (fiqh) consti- 
tués par les commentaires des docteurs de la loi, souvent non traduits. Les seuls auteurs ou commentateurs que nous considérons sont Al-Ghazali et A. Z. AlKayrawani pour l'époque classique, $M$. Hamidullah et A. D. Al-Djazaïri pour la période actuelle.

\section{RÉSURRECTION ET JOUR}

DU JUGEMENT: UN MESSAGE

NOUVEAU PARMI LES ARABES

\section{DU 7E SIECLE}

Nous voulons ici rendre compte de la nouveauté du message islamique parmi les Arabes de l'époque de Mohammed, sans toutefois faire l'historique des notions telles que la Résurrection ou le Jour du Jugement. Dans cette présentation, nous nous basons sur un article de M. Abdesselem (1986: 902), dont nous avons apprécié la clarté. Nous voulons préciser que nous sommes peu en mesure de juger de la justesse de ses affirmations, étant donné notre ignorance de la culture préislamique. Selon lui, l'affirmation de la Résurrection et la menace du Jugement était peu présente chez les Arabes de l'époque, du moins pour ceux qui n'étaient ni chrétiens ni tradition juive. Leur conception de la mort était liée à celle qu'ils avaient de la vie et du monde. La mort était considérée comme le destin du monde animé, qui était alors opposé au monde inanimé. Elle signifiait l'extinction du souffle vital, la séparation de l'esprit et du corps. Vie et mort étaient vues comme immanentes; l'idée même de transcendance était étrangère à leur manière de penser. Enfin, le mort demeurait présent dans l'esprit de la communauté, et était glorifié dans la mesure où il s'était montré glorieux durant son existence. L'existence d'un culte des morts chez les anciens Arabes est une question très débattue, mais il est probable qu'un tel culte ait existé. L'Islam, inspiré par la tradition judéochrétienne, a complètement bouleversé cette vision du monde, de la vie et de la mort. D'abord par l'affirmation d'une transcendance, et de l'opposition Créateur/Création, ensuite par cette idée que c'est le Créateur qui donne la vie et la mort, et que tous les événements terrestres qui semblent être à l'origine de cette vie et de cette mort ne sont en fait que "les intermédiaires à travers lesquels se concrétise la volonté de Dieu " (Abdesselem 1986: 902). L'homme luimême acquiert une dimension nouvelle, liée à cette transcendance de la divinité. Il n'est plus séparé en esprit et corps; dorénavant, il comporte une troisième dimension, qui le fait participer à la transcendance divine, puisque le Coran distingue deux principes vitaux en plus du corps: la nafs (moi pensant) et le ruh (principe de vie qui procède de Dieu et insufflé par Lui). Par l'affirmation de la Résurrection et du Jugement, la mort n'est plus seulement le terme de la vie, mais le début d'une autre vie. Enfin, le mort n'est plus glorifié; l'Islam interdit que le deuil s'éternise, avec l'idée que le mort ne doit pas encombrer l'esprit des vivants. Le culte des morts est tout à fait prohibé, toujours dans l'idée monothéiste que Dieu est le seul objet d'adoration possible.

Nous pouvons mesurer la difficulté qu'a connue Mohammed à convaincre son peuple de la Résurrection par nombre de versets coraniques faisant allusion au scepticisme sur cette matière. Voici une des plus belles réponses du Prophète aux sceptiques:

[...] Et les mécréants dirent:

"Ceci est une chose étonnante.

Quoi! Quand nous serons morts

et réduits en poussière...? Ce

serait revenir de loin! "

Certes, Nous savons ce que la

terre rongera d'eux [de leurs

corps]; et Nous avons un Livre

où tout est conservé. [...]

N'ont-ils pas observé le ciel au-

dessus d'eux, comment Nous

l'avons bâti et embelli; et comment il est sans fissures?

Et la terre, Nous l'avons étendue et Nous y avons enfoncé fermement les montagnes et y avons fait pousser toutes sortes de magnifiques couples de [végétaux], [...]

Et Nous avons fait descendre du ciel une eau bénie, avec laquelle Nous avons fait pousser des jardins et le grain qu'on moissonne, ainsi que les hauts palmiers aux régimes superposés, comme subsistance pour les serviteurs. Et par elle (l'eau) Nous avons redonné la vie à une contrée morte. Ainsi se fera la résurrection. [...] Quoi? Avons-Nous été fatigué par la première créa-

tion?... (Cor. 50; 2-15)

Cette innovation dans la croyance signifie un changement d'attitude fondamental vis-à-vis de la mort. Le Jugement et la Résurrection y occupent une place centrale, ce qui explique l'insistance du message coranique à leur propos. Elle va aussi s'accompagner de modifications au niveau rituel, comme nous le verrons bientôt.

L'AU-DELÀ SELON LE CORAN

Le Coran ne parle pas de la mort en tant qu'événement ponctuel. Ce qui l'intéresse, c'est la vie après la mort, l'au-delà. Celui-ci est principalement centré sur le Jour de la Résurrection et du Jugement. Les deux événements sont liés; il semble en effet que ce soit afin de prendre connaissance de la valeur de ses œuvres que l'homme ressuscite (ex. Cor. 16; 38-39). Ce jour-là, le croyant recevra la révélation du bien et du mal, il se trouvera face à face avec la Vérité. Voici la Sourate 99, appelée Az-zalzala (la secousse), qui décrit le Jour du Jugement:

Quand la terre tremblera d'un violent tremblement, et que la terre fera sortir ses fardeaux ${ }^{2}$, et que l'homme dira: "Qu'a-telle?"

ce jour-là, elle contera son histoire, selon ce que ton Seigneur lui aura révélé (ordonné).

Ce jour-là, les gens sortiront séparément pour que leur soient montrées leurs œuvres.

Quiconque fait un bien fût-ce du poids d'un atome, le verra, et quiconque fait un mal fût-ce du poids d'un atome, le verra.

Cette sourate, malgré sa brièveté, condense les principaux éléments de la croyance au Jour du Jugement. Les corps sont ressuscités; ceci afin que chacun prenne connaissance de la vérité, et de la valeur de chacune de ses actions; ce Jugement se fera de manière individuelle. Il est souvent répété dans le Coran que chacun ne sera rendu responsable que pour ses propres actes (ex. Cor. $6 ; 164$ ou $17 ; 15)$. Le croyant musulman est donc seul devant la mort, définitivement séparé de sa communauté.

Le Jugement est le moment où les actes de chacun sont examinés et où le compte du bien et du mal effectués icibas est rendu. Il est suivi par la séparation des jugés en deux camps, une partie étant destinée au Paradis, l'autre à l'Enfer (description du Jugement ex. Cor. 84;1-12) ${ }^{3}$. Des descriptions de ces lieux sont données à de multiples reprises par le Coran, qui n'est généralement pas favorable à l'intercession, que ce soit de Mohammed lui-même (Cor. 9;80, 74;48 et 26;100). Par contre, il semble que Dieu soit capable de pardonner même à un pécheur, puisqu'il est répété inlassablement que Dieu conduit qui il veut comme il égare qui il veut (ex. Cor. 13;26), ce qui laisse entendre qu'en dernier ressort, Dieu est quand même le seul " Maître du Jugement ", comme il est fait allusion dans la sourate liminaire. Toutefois, nous n'entrerons pas plus avant dans 


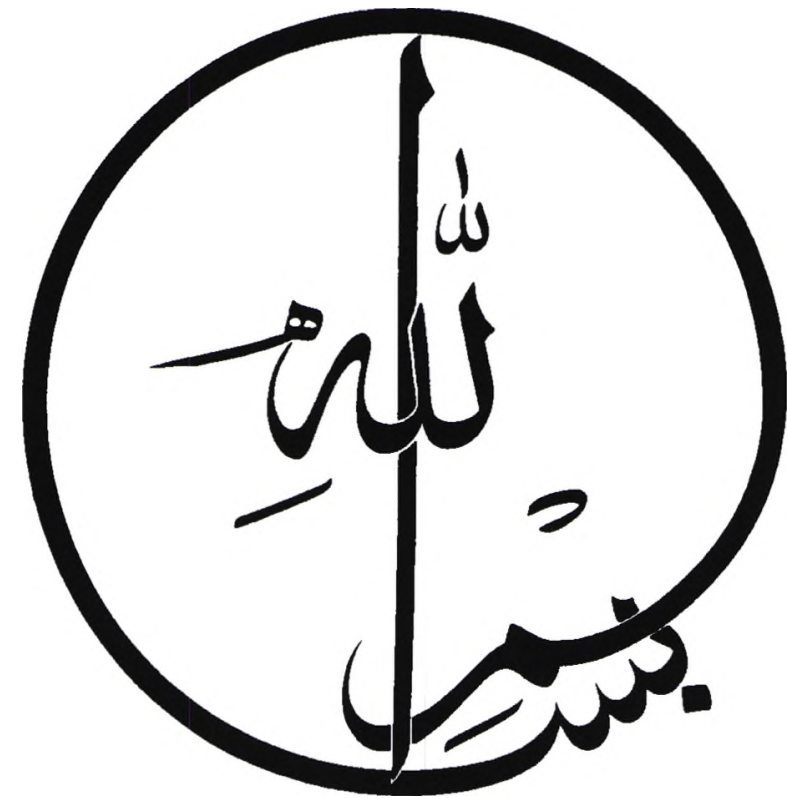

"Au Nom de Dieu "

cette discussion, qui touche à la question de la prédestination. Si le Jour du Jugement, le Paradis et l'Enfer sont décrits assez précisément, le Coran reste en revanche obscur sur le destin de l'homme immédiatement après sa mort. Certaines écoles ont supposé que "l'homme après la mort tombait dans un état d'inconscience pour ne plus retrouver ses sens qu'au moment de la résurrection 》 (Sourdel 1961: 187). D'autres écoles (les asharites et certains mu'tazilites) pensent que les morts accèdent directement au paradis ou à l'enfer.

\section{PERSPECTIVE DES HADITHS:}

\section{LE CHÂTIMENT DE LA TOMBE}

C'est peut-être à cause de ce vide coranique concernant le temps qui sépare la mort de la résurrection que l'image du " châtiment de la tombe " a été développée par plusieurs traditionnistes (auteurs des recueils de Hadiths). Il s'agit là d'une innovation complète de leur part, puisqu'il n'en est fait aucune mention dans le Coran. Il s'agit en fait d'un préjugement, effectué directement après l'ensevelissement du défunt, par les deux anges Munkar et Nakir (présents dans le Coran, mais au Jour du Jugement) qui questionnent le mort d'une manière effrayante et à qui celuici doit répondre en attestant de sa foi. Voici une des descriptions de ce châtiment véhiculées par les Hadiths:
L'Envoyé de Dieu a dit: "Quand le mort a été descendu dans sa tombe, que ses amis se sont éloignés et qu'il entend encore le claquement de leurs sandales, voici qu'il voit venir à lui deux anges qui le font dresser sur son séant et lui disent au sujet de Mahomet: "Que disais-tu de cet homme? "Si le mort est un vrai croyant il répondra: "Je confesse qu'il est le serviteur et l'Envoyé de Dieu. " Alors les anges lui diront: "Regarde la place que tu aurais occupée en enfer. En échange Dieu t'a assigné une place dans le paradis. " Et l'homme verra à la fois les deux places [...]. Quant à l'hypocrite ou au mécréant, à la question qui lui était posée [...], il répondra: "Je ne sais trop; je répétais ce que tout le monde disait. " (AlBokhari 1977: 444)

La scène est ensuite suivie d'un préjugement, le bien-être pour le croyant qui aura bien répondu et la souffrance pour le mécréant ou l'hypocrite, et ceci jusqu'au Jour du Jugement ${ }^{4}$. Cette croyance, bien que condamnée par les adeptes du mu'tazilisme (école de pensée rationaliste du $9 \mathrm{e}$ s. ap. J.-C.) de même que par les kharijites et certains courants shî'ites, s'est largement popularisée. Elle est d'ailleurs réaffirmée par Al-Ghazali au 12e siècle (1878: 22).
Aujourd'hui encore, elle reste souvent citée par les croyants, et tient une grande place dans leur imaginaire.

\section{LA CROYANCE EN L'AU-DELÀ EST UN ARTICLE DE FOI}

La croyance en l'existence de l'audelà constitue le cinquième article de foi de la religion musulmane, qui en comprend cinq ou six, selon les traditions. Voici le célèbre Hadith dit de Gabriel, rapporté par Al-Bokhari:

Le Prophète s'étant un jour mon-

tré en public au peuple, un

homme vint le trouver et lui dit:

"Qu'est-ce que l'iman (la foi)? -

C'est, répondit-il, croire en Dieu,

à ses anges, à l'autre vie, aux

prophètes et à la Résurrection.

[...] » (1977: 28)

Ailleurs, on trouve d'autres listes d'articles de foi, mais elles ne different pas fondamentalement de celle-ci. Toutefois, une autre version du hadith de Gabriel, rapportée par An-Nawawi, ajoute un sixième article de foi, qui est la croyance en la prédestination. Cela démontre à quel point la croyance en l'au-delà, dans la Résurrection et au jour du Jugement tient une place importante dans le corpus de la croyance islamique:

[...] Informe-moi maintenant, dit

l'étranger, du contenu de la foi

en Islam. - " C'est, dit le

Messager d'Allah, de croire en

Allah, en Ses Anges, en Ses

Livres, en Ses Messagers, enfin

au Jour du Jugement dernier et

au Destin préétabli, pour le

meilleur comme pour le pire. »

(1980: 22)

Le fait que la croyance en l'au-delà fasse partie du corpus de base de la foi islamique signifie que la croyance en la vie après la mort est indissociable du reste de la foi musulmane. Elle s'insère de manière cohérente dans la conception du monde propre à l'Islam. C'est ce que dit aussi W.C. Chittick, lorsqu'il affirme que la croyance en la vie après la mort constitue un des trois principes de base de la pensée théologique musulmane. Ces trois principes sont l'Unité de Dieu (tawhîd), la prophétie (nubuwa) et l'eschatologie (mawt), c'est-à-dire toutes les questions touchant à la vie future. Pour comprendre la conception de la mort dans l'Islam, il faut donc passer par la conception de Dieu et du sens de la Création. Le tawhîd ne veut pas seulement dire qu'il n'y a de Dieu que Dieu, mais aussi que toute chose dans l'univers provient d'une source unique et est inextricable- 
ment liée à elle. La prophétie ne vient pas seulement rappeler que Dieu est unique mais aussi que l'homme est inséparable de son Créateur et de son origine. C'est parce que l'homme est créature de Dieu qu'il lui faut suivre ses conseils pour profiter au mieux de son existence et la vivre avec le plus de signification. Ce lien de la créature au Créateur entraîne l'idée que toute chose vient de Dieu et retournera à Lui (Chittick 1969: 125-6). La mort fait ainsi partie intégrante d'un processus logique; plus encore, la vie ne prend son sens que dans la mort, puisque celle-ci, en plus d'un retour au point d'origine, marque l'aboutissement de la vie d'ici-bas, à savoir la découverte de la Vérité, la connaissance du bien et du mal, et le jugement des actes et des pensées de la vie de ce monde.

\section{AUTRES CONCEPTIONS DE L'AU-DELÀ DANS L'ISLAM}

Nous avons exposé ci-dessus la conception de l'Islam sunnite majoritaire. Indiquons simplement qu'il existe dans plusieurs courants spiritualistes ou mystiques de l'Islam, des conceptions de l'au-delà qui leur sont propres. La voie mystique musulmane, le soufisme, à l'instar de toutes les mystiques, intériorise les rites de la religion officielle, et vit sa croyance comme un sentiment intérieur et permanent de la présence divine. Le corps du soufi possède en lui tous les atomes de l'univers. L'homme est ainsi une synthèse ou un miroir de tous les mondes, visibles et invisibles. Selon Bianu, le paradis et l'enfer ne seraient pas extérieurs à lui mais intérieurs. C'est l'homme luimême qui se crée son paradis et son enfer selon son degré d'élévation spirituelle. Ce paradis et cet enfer sont en lui (Bianu 1981: 202-3). De même, certains courants spiritualistes proposent d'autres formes de croyance en l'audelà5. Il s'agit par exemple des Ismaïliens, des Bektashi, des Druzes, des Nutsaïris ou des Ahl-e Haqq (les Gens de la Vérité), qui " admettent à des degrés divers la migration des âmes »(Bianu 1981: 204-5). Selon les Ahl-e Haqq, une vie ne saurait suffire à atteindre la perfection. Chaque âme a reçu originellement un certain nombre d'enveloppes humaines, qu'elle va devoir habiter avant de pouvoir rejoindre l'essence divine.

\section{CONCLUSION}

La conception de la mort dans l'Islam nous semble tenir une place prédominante au niveau de la foi. Elle ne représente ni une cassure, ni une abomination. Elle s'insère dans le cycle voulu par Dieu de la naissance-mort-résurrection. D'ailleurs, l'Islam populaire compare volontiers la période qui sépare la mort de la résurrection aux mois de gestation dans le ventre de la mère. À l'intérieur de ce cycle, la mort permet le retour de la créature au Créateur, en même temps qu'elle donne un aboutissement signifiant à la vie d'ici-bas. Le mot qui résume peut-être le mieux cette conception est celui de "continuité ". Si la mort donne sens à la vie, cela veut dire aussi que la mort parle de la vie, que lorsque la mort est évoquée, c'est du même coup aussi de la vie que l'on parle. L'idée de la mort donne à l'homme une tâche pour cette vie-ci. Car c'est durant toute sa vie qu'il va préparer sa mort, qu'il en soit conscient ou non.

\section{DU CÔTÉ DES RITES}

Nous nous attacherons à montrer l'importance de chaque rite, son degré d'obligation donné par les Hadiths ou développé par les commentateurs. Ce degré d'obligation au niveau des textes ne correspond pas toujours à sa popularité auprès de la population musulmane. Chaque communauté utilise à sa façon le corpus religieux et procède à des réaménagements éventuels. En effet, l'intensité d'une pratique ne reflète pas forcément la place qu'elle tient dans la loi. Nous évoquerons aussi la question de la dimension socio-historique du rite funéraire dans l'Islam. Puis nous isolerons trois caractéristiques qui nous semblent le mieux résumer l'" esprit » du rite funéraire islamique. Ensuite, nous exposerons l'idée de continuité de la vie à la mort qui nous semble être présente dans le rite funéraire islamique. Enfin, nous nous demanderons à qui sont destinés les différents rites? Nous verrons que si un certain nombre de rites s'adressent au défunt, d'autres ont une valeur morale évidente, et semblent plutôt destinés aux survivants.

Précisons que les rites sont obligatoires dès la puberté, soit 13-14 ans, selon les cas $^{6}$. En-dessous de cet âge, la pratique n'est que recommandée.

\section{LES RITES FUNÉRAIRES SELON LES HADITHS}

Le Coran donne peu de précisions concernant le rite funéraire. L'inhumation du mort est la seule indication rituelle précisée par celui-ci. "N'avons-Nous pas fait de la terre un endroit les contenant tous, les vivants ainsi que les morts? "(Cor. 77;25-6).
Une autre évocation de l'inhumation se trouve à la Sourate 80, v. 21 . La terre est donc la seule destination possible du défunt pour un musulman. Par conséquent, l'incinération est absolument proscrite, de même que toutes autres formes de sépulture.

Ce sont surtout les Hadiths auxquels on se réfere en matière de rite funéraire. En effet, tout un ensemble de chapitres sont consacrés aux pratiques et aussi aux attitudes qu'il s'agit d'avoir en cas de décès d'un membre de la communauté, exposées de manière très détaillée. Le corps du défunt est pris en charge immédiatement après sa mort, s'il n'a pas été possible pour les proches d'assister aux derniers instants de sa vie. En effet, il est considéré comme très important de réciter une dernière fois la shahada (profession de foi) à l'oreille du mourant, au moment où luimême est trop faible pour le faire. Une des raisons de ce rite serait cette croyance au châtiment de la tombe que nous venons d'évoquer. Le fait de réciter la profession de foi à l'oreille du mourant doit l'aider à s'en rappeler au moment où les deux anges l'interrogeront dans la tombe (Sourdel 1961: 200). Mais il se peut aussi qu'elle ne soit qu'une préparation au Jour du Jugement lui-même, puisqu'un hadith affirme que chacun apparaîtra lors de sa résurrection dans le même état que lorsqu'il meurt (Bianu 1981: 197-8). Ce qui expliquerait aussi pourquoi le musulman meurt l'index levé, geste qu'il a eu tout au long de sa vie lors des prières, et qui signifie la croyance en l'unicité de Dieu. L'affirmation de la Résurrection des corps, qui revient à de très nombreuses reprises dans le Coran (nous avons vu Cor. 50; 2-15), explique l'interdiction absolue d'exhumer un corps.

Les détails concernant la manière d'accomplir les divers rites funéraires abondent dans les Hadiths. Il est toutefois possible de retracer une sorte de scénario minimum composé de trois pratiques, respectées par chaque tradition et chaque commentateur. Il s'agit: de la toilette du corps (et de son emmaillotement dans le linceul), de son inhumation à même la terre, (le visage tourné vers la Mecque) et de la prière sur le mort. Les Hadiths donnent encore beaucoup de détails quant au nombre de pièces d'étoffe tenant lieu de linceul, leur couleur, sur la manière de laver le corps, de tresser les cheveux de la défunte, etc. Là-dessus, chaque peuple a ajouté quelques pratiques relevant peut-être de sa propre culture pré- 
islamique ou développées parallèlement à la tradition islamique. Nous n'insisterons pas sur les différentes variations existant d'une école juridique à l'autre. Les malikites interdisent de porter le mort à la mosquée pour la prière et les avis diffèrent sur la question de laver ou non le corps d'un martyr (Lammens 1943: 118). Les principaux rites sont pourtant communs aux quatre écoles.

Voici un descriptif d'une cérémonie de funérailles: on récite sur le mort la sourate Ya Sin (Cor. 36), considérée comme le cœur du Coran. Puis on procède à la toilette du mort et à sa mise au linceul. Vient ensuite le cortège funèbre, qui se fait à pied: on transporte le corps à la mosquée (ou directement au cimetière, selon les écoles) pour une prière qui se fait debout (on ne se prosterne pas devant un mort); puis le corps est porté au cimetière, où il est enseveli à même la terre, le visage tourné vers la Mecque. Invocations, rappel des actions du défunt par l'imam ou un proche tandis que la terre est répandue sur le corps. Puis les gens qui ont assisté à l'enterrement présentent leurs condoléances à la famille du défunt, soit directement au cimetière, soit à son domicile. Durant la nuit qui suit l'ensevelissement, on récite le Coran divisé en trente parties. Au troisième jour (le deuil dure trois jours, sauf pour une femme ayant perdu son époux), certains vont visiter la tombe. C'est souvent à ce moment-là que les femmes vont au cimetière pour la première fois, puisque certains hadiths leur déconseillent d'assister au cortège funèbre le jour de l'inhumation ( AlBokhari 1977: 413).

\section{LE DEGRÉ D'OBLIGATION}

\section{DES RITES FUNÉRAIRES}

L'Islam ne distingue pas le sacré du profane. Les funérailles tiennent la place d'un rite de passage, le plus important de l'existence d'un musulman. En effet, la mort revêt davantage de signification religieuse que la circoncision ou le mariage dans l'Islam.

Pourquoi parler de degré d'obligation? Le droit musulman (figh) prévoit toute une gamme de niveaux d'obligation du rite. Les versets coraniques ou les Hadiths n'étant pas clairs à ce propos, il a fallu que les juristes des premiers siècles de l'Islam établissent euxmêmes cette échelle, selon les règles de l'interprétation coranique. Ainsi, ils ont distingué cinq niveaux d'obligation. L'interdit (haram) s'oppose à l'obligatoire (wâjib), le déconseillé (makruh) au conseillé (mustahab); reste la cinquième catégorie, (ce qui est permis ou naturel, hallal, mubah) dans laquelle on range tous les gestes qui ne font partie d'aucune autre catégorie, comme par exemple celui de boire un verre d'eau.

Il est difficile d'établir avec précision le degré d'obligation des différents rites funéraires, les écrits des commentateurs n'ayant pas été traduits de la langue arabe. Selon D. Sourdel, les juristes se sont accordés pour rendre obligatoires les quelques rites suivants: il s'agit de la toilette du corps, de sa mise en terre et de la prière sur le mort (1996: 586). Tout le reste est de l'ordre du conseillé. Cela pose la question de l'interprétation de la loi coranique. Comment les juristes vont-ils décider du caractère obligatoire de tel ou tel rite? Il semble que certaines interprétations juridiques soient religieuses, alors que d'autres seraient motivées davantage culturellement ou socio-politiquement. La séparation des corps musulmans et non-musulmans dans les cimetières, estimée comme obligatoire par une bonne partie de la population musulmane en Suisse, n'est commandée ni par le Coran ni par les Hadiths. Par contre, les écoles de droit ont prescrit par la suite que les musulmans ne pouvaient être enterrés avec des nonmusulmans (Pieri 1995: 60). Faut-il considérer ces développements juridiques comme faisant partie du corpus religieux? Tout dépend de la définition que nous en donnons.

En tous cas, on constate une certaine souplesse dans la présentation des pratiques par les Hadiths. Souplesse, dans le sens d'abord que les Hadiths ne limitent pas le rite à une forme unique, mais en donnent souvent plusieurs versions, dont chacune est valable religieusement et légalement. Ainsi du nombre de pièces d'étoffe requises pour le linceul. Certains hadiths parlent de trois pièces d'étoffe, d'autres de deux. Même en ce qui concerne la toilette du mort, les commentateurs font preuve de souplesse, en n'imposant pas une façon de faire. A. D. Al-Djazaïri, une des principales références sunnites actuelles, admet dans son célèbre ouvrage " La voie du musulman »: "Il serait suffisant de verser de l'eau dessus de sorte qu'elle touche toutes les parties du corps, mais la manière préférable et complète est... " (1986: 295). Suit toute une liste de précisions. Cet auteur respecte en cela l'esprit de ses prédécesseurs, puisque A.Z. Al-Kayrawani, au 4 e s. de l'Hégire, fait preuve de la même souplesse: «Il n'y a point de limitation précise pour le lavage du mort. Ce que l'on doit avoir en vue c'est [simplement] de le rendre propre " (1952: 105).

Souplesse ensuite, dans le sens où la tradition elle-même (les Hadiths) prévoit la possibilité d'adapter le rite à une situation réelle. Le chapitre 28 évoque par exemple le cas où les compagnons du Prophète n'avaient qu'un manteau pour couvrir le corps d'un défunt (AlBokhari 1977: 409-13). La tradition prévoit également qu'en cas de guerre ou d'hécatombe, deux corps puissent être enterrés ensemble dans la même fosse, ce qui est déconseillé en temps normal (Al-Bokhari 1977: 433). L'Encyclopédie de la jurisprudence islamique rapporte même qu'en cas de décès en mer, les juristes autorisent à jeter le corps par-dessus bord, si la côte ne peut pas être atteinte dans les deux jours (1983: 381). Cette dernière affirmation prouve que l'adaptation de la loi à la réalité humaine peut se faire sur une échelle très large et toucher même les rites les plus obligatoires.

En résumé, nous pouvons affirmer que l'Islam propose une pratique parfaite, mais se contente aisément d'une forme moins complète, pourvu que les quelques impératifs soient respectés. Certes, il semble que les simplifications rituelles soient surtout justifiées en cas de nécessité. Cette notion de "nécessité » existe d'ailleurs en juridiction. Il s'agit de la darura (nécessité), qui permet une modification ou même l'abandon d'un rite. Cette notion de darura a pour corollaire l'ijtihâd, qui est la possibilité d'interpréter le texte coranique, possibilité accordée à tout croyant connaissant les Textes religieux.

En définitive, il semble que la forme rituelle soit négociable, mais surtout dans la mesure où elle respecte l'esprit du rite. L'esprit prime sur la lettre. Cet esprit, c'est avant tout l'humilité et la sobriété devant cet événement qui dépasse la volonté humaine. D'ailleurs, sur le plan socio-historique, on remarque que c'est davantage l'esprit du rite que le rite lui-même qui a été modifié avec l'apparition de l'Islam. M. Abdesselem affirme en effet que les principaux rites cités précédemment sont repris des rites funéraires arabes préislamiques. La toilette des morts, le linceul et l'enterrement se faisaient déjà. Mais l'Islam introduit la prière sur le mort, qui " confère à toute la cérémonie funéraire une signification radicalement différente. Ce n'est plus une glorification du mort mais une implora- 
tion de la miséricorde divine. À une éthique d'exaltation, l'Islam a substitué une morale d'humilité. " (Abdesselem 1986: 903). C'est dans ce même esprit qu'il va interdire certaines pratiques comme les lamentations ou les offrandes, courantes à l'époque du Prophète.

\section{L'ESPRIT DU RITE FUNÉRAIRE ISLAMIQUE}

\section{L'humilité}

En effet, l'Islam de l'époque de Mohammed, comme toute religion à ses débuts, a cherché à se démarquer des autres croyances présentes. Toute religion s'inspire de son environnement socioculturel, et toute religion cherche aussi à s'émanciper de cette influence, dans sa volonté d'affirmer clairement sa différence ou du moins sa spécificité. Chaque religion naissante, même révélée, doit trouver son identité propre. Celle de l'Islam, nous l'avons déjà évoqué, se situe entre autres dans son affirmation d'une transcendance, et dans sa soumission à cette transcendance. Dans le cas de l'attitude devant la mort, l'Islam va ainsi introduire un esprit d'humilité et de résignation qui n'existaient pas auparavant, du moins pas du même type. Avec l'Islam, ce n'est plus devant le mort qu'on va s'agenouiller, mais toujours devant Dieu? cendance de Dieu et son unicité constituent la base de laquelle tout découle. L'homme, qu'il soit vivant ou mort, reste créature de Dieu. Il est totalement dépendant de lui et lui doit tout: la vie, la mort, la résurrection. Dès lors, le mort n'est pas plus sacré que le vivant, et il ne possède aucune qualité surhumaine ou occulte.

C'est ainsi qu'une première interdiction va être formulée: celle de construire des lieux de culte sur les tombes. Mohammed va se montrer intransigeant sur cette matière. Voici le chap. 71 des Hadiths recueillis par M. I. Al-Bokhari:

'Aïcha a dit: "Pendant que le Prophète était très souffrant, certaines de ses femmes parlèrent d'une église qu'elles avaient vue en Abyssinie et qu'on appelait (église de) Maria. Oum-Salama et Oum-Habiba, qui étaient allées en Abyssinie, vantaient la magnificence de cette église [...]. Le Prophète leva la tête alors et dit: "Lorsqu'un saint personnage d'entre eux vient à mourir, ces gens-là bâtissent sur sa tombe un oratoire [...]. Ces gens-là sont les pires des êtres créés aux yeux de Dieu. " (1977: 432)

Ce sentiment de résignation devant le décret divin imprègne l'ensemble des rites funéraires islamiques. Chaque geste exprime la nécessité de ne montrer aucun signe d'opposition ou de plainte devant la volonté divine. Tout d'abord, l'accomplissement des rites doit avoir lieu le plus vite possible. Un hadith dit en effet que le mort doit aller le plus rapidement possible au lieu qui l'attend (Al-Bokhari 1977: 424-5). Le corps doit être rendu au plus vite à son Créateur, auquel il doit retourner. C'est pour cela que les juristes ont décidé qu'un défunt devait être enterré le jour même, sinon le lendemain au plus tard. Par cette rapidité, on exprime une acceptation du plan de Dieu et l'idée que les gestes des croyants doivent aller dans le sens de cette volonté.

Outre la rapidité d'inhumation, l'humilité s'exprime également dans le fait que l'homme ne peut absolument pas intervenir sur le corps du défunt, car en tant que création divine, ce corps est sacré. C'est pourquoi il n'est pas pensable de brûler un corps ou simplement de le maltraiter. Le corps du défunt doit être manié avec la plus grande douceur, lors de la toilette ou du transport au cimetière. Selon AlGhazali, il semble que le défunt continue à souffrir des mauvais traitements endurés à ce moment-là: "Un autre encore apparut [en songe] et on lui dit: "Qu'est-ce que Dieu t'a fait? " Il répondit: " Le baigneur qui m'a lavé après ma mort m'a porté avec violence et un clou qui était dressé dans la baignoire m'a écorché. J'en souffre encore. » (1878: 24-5). C'est également dans ce sens d'humilité que vont les interdictions répétées des lamentations sonores et plaintives, comme si celles-ci signifiaient un manque de résignation. Voici un de ces chapitres:

'Abdallah a dit: "Le Prophète s'est exprimé ainsi: "Ils ne sont pas des nôtres ceux qui se frappent les joues, qui déchirent les encolures de leurs vêtements et qui profèrent des invocations de l'époque antéislamique. " (Al-

Bokhari 1977: 418)

La tradition ira même jusqu'à interdire que le deuil dure plus de trois jours, sauf pour une femme ayant perdu son époux. Le deuil dure alors plus de quatre mois, et s'accompagne de l'obligation pour cette femme de rester célibataire (Al-Bokhari 1977: 413). Cette brièveté du temps de deuil signifie là encore une volonté de ne pas s'appesantir sur la perte d'un proche, puisque c'est Dieu qui a voulu sa mort.

Enfin, citons simplement les paroles du Prophète "lui-même ", tirées d'un hadith d'Al-Bokhari: "Le Prophète fit répondre [...] que tout ce que prenait Dieu et tout ce qu'il donnait lui appartenait; que chacun avait son terme fixé par Dieu, qu'elle [sa fille] devait donc se résigner, et que Dieu lui en tiendrait compte. » (1977: 415)

\section{La sobriété}

L'ensemble du rituel musulman est caractérisé par une certaine souplesse, nous l'avons vu. Il est souvent précisé que si une pratique ne peut être accomplie, les effets n'en sont pas dramatiques pour le croyant. La devise est la suivante: pas de contrainte en religion (ex. Cor. 2;256). D'autre part, la " simplicité » représente une sorte d'impératif formel. Par ce terme de simplicité, nous entendons la "sobriété " du rite. Cette sobriété n'est pas seulement un état de fait, elle doit être préférée par le croyant. Par exemple, les dépenses excessives sont très mal considérées, voire tout à fait interdites, par exemple chez M. Hamidullah, qui proscrit absolument toute dépense pour les tombes (1963: 205). alors que d'autres auteurs se contentent de conseiller la simplicité en cette matière. Le mort est nu dans son linceul. Le linceul lui-même ne doit pas être de beau tissu. Aucun artifice ou décoration ne l'accompagne. Au cimetière, la tombe doit être la plus simple possible. On marque l'endroit d'une pierre dressée, où l'on écrit seulement le nom de la personne enterrée, mais sans ajout de texte. La pierre elle-même ne doit pas être d'un matériau précieux comme le marbre 8 . À l'origine, les tombes, qui n'étaient pas construites en dur, finissaient par s'éroder et disparaître, fondues dans le paysage. Cette exigence formelle est l'expression visible de la conception musulmane de la mort, qui comporte elle-même cette attitude de résignation et de sobriété. Mais elle est aussi plus pragmatique: l'argent dépensé pour un mort est une 
perte. Les vivants en ont davantage besoin. Une telle idée se trouve aussi dans les Hadiths (Al-Bokhari 1977: 449). Ce pragmatisme se retrouve dans le fait qu'on ne s'embarrasse d'aucun acte superflu. Pas de Coran posé au chevet du mort, pas de fleurs sur la tombe, rien qui ne soit efficace, dans l'optique du salut futur.

\section{La pureté}

L'impératif de pureté est omniprésent dans la religion islamique. Les interdits alimentaires, les ablutions avant chaque prière, l'interdiction d'avoir des rapports avec une femme pendant les menstruations découlent de cet impératif. Au moment de la mort, ce point est traité avec une attention particulière. Il s'agit en effet d'un moment critique pour le croyant, puisque c'est dans l'état où il était à sa mort qu'il va apparaître à son Créateur. C'est ainsi que la toilette du mort se trouve être un des moments cruciaux du rite funéraire. Précisons que cette toilette comporte entre autres les ablutions, pratiquées tout au long de la vie, et qu'on répète une dernière fois. De même, le mourant se doit de prononcer une dernière fois la profession de foi, et lève son index en signe d'allégeance au tawhîd, l'unicité de Dieu, qui représente un principe central dans l'Islam.

\section{DE LA VIE À LA MORT,}

UNE CONTINUITE

Tous ces rites donnent l'impression d'une continuité entre la vie et la mort, dans l'Islam. Il nous semble en effet que les rites funéraires reflètent les pratiques des vivants: la toilette du mort est précédée des ablutions, un geste que l'on répète tout au long de sa vie; le linceul, dans le meilleur des cas, est le doivent s'accomplir dans la rapidité, la mort ne fait l'objet d'aucune mise à l'écart. La toilette du mort, par exemple, n'est pas réservée à des personnes dont c'est la fonction de le faire, mais tout un chacun peut se trouver amené à le faire un jour, pour un membre de sa famille, proche ou éloignée. La mort est incluse dans la vie, elle en fait partie. Il nous semble que ce point reste une réalité pour la communauté musulmane actuelle, et qu'il n'est pas seulement un idéal présent dans les textes.

\section{LA MORT DE L'AUTRE COMME ENSEI- GNEMENT}

Cela nous rappelle que la mort peut être vue selon deux angles: la mort de l'autre et la mort de soi. Les moralistes estiment que la mort de l'autre est une occasion de rappel à l'ordre pour tous les survivants. Il est intéressant de noter alors que certains rites sont destinés surtout à ces derniers, à la communauté des vivants qui a perdu l'un de ses membres. La mort de l'un permet au reste de la communauté de méditer sur cet événement qui va toucher chacun. Al-Ghazali consacre une partie de sa "Vivification des sciences de la foi » à cette valeur morale de la mort. "Les obsèques sont un sujet de méditation pour l'homme clairvoyant, un avertissement pour les insouciants. " (1955: 436). Al-Ghazali encourage donc toutes les pensées et pratiques qui donnent l'occasion au croyant de se rappeler son caractère mortel. Du côté de la pensée: "Il faut se libérer de toute autre pensée que celle de la mort; méditer sur celle d'autrui... » (1955: 433). Quant aux pratiques, Al-Ghazali conseille la visite des tombes. N'importe quelles tombes, d'ailleurs, et

OUTRE LA RAPIDITÉ D'INHUMATION, L'HUMILITÉ S'EXPRIME

ÉGALEMENT DANS LE FAIT QUE L'HOMME NE PEUT ABSOLUMENT PAS

INTERVENIR SUR LE CORPS DU DÉFUNT,

CAR EN TANT QUE CRÉATION DIVINE, CE CORPS EST SACRÉ.

tissu dont s'est revêtu le défunt lors de son pèlerinage à la Mecque; les prières récitées sont des versets coraniques qui ne sont pas réservés à cette occasion mais que l'on répète souvent au cours d'une existence; l'attitude du mourant, récitant la shahada (profession de foi) et l'index levé, est une répétition finale de l'attitude de tout croyant de son vivant. Enfin, même si les funérailles te, car propice au développement de cultes des morts, pratique absolument prohibée par le Prophète lui-même. La tradition a peut-être préféré mettre l'accent sur le lien entre les morts et les vivants, et sur la valeur morale de ces visites des cimetières, qui non seulement ont été maintenues, mais même encouragées, et ceci par les plus grands penseurs de l'Islam.

Cette nécessité de se rappeler qu'on va mourir est liée à l'idée que les plaisirs d'ici-bas sont vains et que seule importe la vie future. Cette idée n'est d'ailleurs pas due aux moralistes, puisqu'elle est déjà très présente dans le Coran (ex. Cor. 6;32). Elle est souvent contrebalancée par l'invitation à profiter des bienfaits mis à notre disposition par le Créateur (ex. Cor. 5;87-8); mais cette jouissance des plaisirs de l'existence doit se faire avec modération (ex. Cor. 7;31-2). L'important étant de ne pas perdre de vue le fait que la vie d'ici-bas n'est qu'une préparation en vue de la vie future, du Jugement et de la rétribution. La vue ou la pensée de la mort rappelle ainsi au croyant que sa vie lui vient de son Créateur et que celui-ci peut l'interrompre à n'importe quel moment. Qu'il y a donc perpétuelle urgence, si l'on veut arriver devant lui dans un état de pureté suffisante.

Mais ce lien des vivants avec la mort ne s'exprime pas seulement au niveau moral. Sur le plan strictement religieux, le croyant qui remplit ses devoirs envers le défunt sera récompensé dans l'au-delà.

L'Envoyé de Dieu a dit:

"Quiconque suivra le convoi funèbre d'un musulman comme acte de foi et par pur amour de Dieu, puis accompagnera le corps jusqu'à l'achèvement des prières et la mise en terre, recevra une double part de récompense, chacune de ces parts équivalent en poids à la montagne de Ohod. Celui qui s'en ira avant que le corps ne soit mis en terre ne profitera que de la moitié de cette récompense. »( $\mathrm{Al}$-Bokhari 1977: 26)

Les vivants sont donc liés aux morts par des obligations, des devoirs. On dit aussi que le mort a des droits sur le vivant. Le chapitre 2 des Hadiths d'AlBokhari évoque les devoirs qu'un musulman a envers un autre musulman:

Un musulman a cinq devoirs à accomplir à l'égard d'un autre musulman: lui rendre son salut, 
le visiter quand il est malade, suivre son convoi funèbre, accepter son invitation et lui dire:

"Dieu te fasse miséricorde "

quand il éternue. (1977: 402)

De même, la prière sur le mort est considérée comme un devoir du croyant envers le défunt. Voici ce qu'en dit A. D. Al-Djazaïri: il s'agit d'une " responsabilité commune, incombant à chaque membre de la communauté. Son accomplissement par un fidèle décharge le reste de la population." (1986: 297). Le même auteur estime souhaitable pour ceux qui assistent à l'enterrement de "demander pour le mort le pardon, d'implorer Dieu, de l'affermir [le défunt] dans son interrogatoire»(1986: 300).

On se rend compte alors de la relation complexe qu'entretiennent les Textes et la tradition. Car alors que le Coran semble affirmer la nullité des intercessions des vivants pour le mort, les commentateurs réintroduisent cet usage de la prière pour la rémission des péchés. Al-Ghazali lui-même affirme leur efficacité (1955: 437). A. Z. AlKayrawani (4e/11e s.) rapporte un exemple d'invocation sur le mort:

$\mathrm{O}$ mon Dieu! [...] Nous venons à

toi en intercesseurs en sa faveur.

Fais droit à notre intercession

pour lui.

O mon Dieu, garde-le de toute

faiblesse lors de l'interrogatoire

du tombeau, préserve-le du châ-

timent de l'enfer! (1952: 111).

Les invocations profitent donc à la fois au mort et à celui qui l'accomplit. L'affirmation de ce lien entre les vivants et les morts semble entrer en contradiction avec ce que nous disions précédemment, à savoir que le croyant est définitivement seul devant la mort. Le Jugement est en effet individuel, et la mort marque le moment de coupure entre un individu et la société. L'insistance mise sur l'accompagnement du mourant puis du défunt signifie peut-être une volonté d'adoucir cette séparation en réaffirmant l'unité de la communauté, au moment même où celle-ci perd l'un de ses membres.

\section{ISLAM CORANIQUE ET ISLAM POPULAIRE}

Cela nous amène à évoquer la question de la relation entre un Islam coranique (ou normatif) et l'Islam tel qu'il est ensuite pratiqué par la majorité des fidèles. Nous avons présenté les croyances et les rites tels qu'ils sont donnés par les Textes fondateurs de l'Islam, le Coran et les Hadiths (ou
Sunna). Mais nous n'avons pas traité du problème de leur application par la communauté des croyants. Il est clair pourtant que certaines pratiques se trouvent en contradiction par rapport aux Textes. Nous citerons seulement deux exemples de ces pratiques populaires contraires à l'orthodoxie: celle des pleureuses, et celle du culte des saints, toutes deux probablement héritées de l'époque anté-islamique, et qui sont demeurées très vivaces dans la population, malgré l'interdiction répétée du Prophète (qui, nous l'avons vu, interdit aussi bien les lamentations exagérées que le culte des morts).

\section{Notes}

1 Ce dernier partage en effet les principaux rites funéraires avec les quatre écoles sunnites.

2 Le mot "fardeaux " fait ici référence aux corps enterrés

3 Il existe également un troisième lieu, l'a'raf (ou les a'râf), mentionné dans le Coran (Cor. 7, 46-47), qui serait, selon les commentateurs, soit un simple voile ou une muraille séparant le paradis de l'enfer, soit un troisième lieu dans lequel sont placés ceux qui n'ont leur place ni en enfer ni au paradis, ou placés là dans l'attente d'aller au paradis.

4 À propos du lien entre le corps et l'âme immédiatement après la mort, la pensée musulmane propose deux grandes lignes divergentes (la ligne traditionnelle, spécialement asharite, et la ligne spiritualiste des shî'ites et de certains mu'tazilites). Voir à ce propos Ghazali.

5 De même que quelques auteurs sunnites isolés. Voir Al-Shahrastani, Les religions et les sectes, Leuven: Peeters, Paris, 198693 (l'auteur a vécu au 6e/12e siècle)

6 À l'apparition des caractères sexuels secondaires, des menstruations, de la capacité de procréer.

7 Nous parlons bien ici de l'Islam coranique. Les pratiques populaires ne respectent pas toujours ce qui est prôné par les textes (ex. les pélerinages sur les tombeaux des saints).

8 Là encore, précisions que nous parlons de l'esprit du texte coranique. La pratique est plus nuancée

\section{Bibliographie}

Sources islamiques:

Le Saint Coran et la traduction en langue française du sens de ses versets, Prés. gén. des Dir. des Recherches scient. islam., Arabie Saoudite, 1990 (1410 H.). Le Coran, essai de trad. J. Berque, Paris, Albin Michel, 1995.

M. Ibn I. AL-BOKHARI, Les traditions islamiques, trad. O. Houdas et W. Marçais, t. I, Paris, Maisonneuve, 1977.

A. Z. Y. Ibn S. AN-NAWAWI, Quarante Hadiths, trad. S. Mazigh, Tunis, SudÉditions, 1980

M. AL-GHAZALI, Vivification des sciences de la foi (Ih'ya), an. G.-H. Bousquet, Paris, Max Besson, 1955
M. AL-GHAZALI, Revivification des sciences de la foi (Ih'ya), trad. A. Moussali, Alger, Entr. nation. du Livre, 1985.

M. AL-GHAZALI, La Perle précieuse, trad. L. Gautier, Paris, Londres, Maisonneuve \& Cie, Williams \& Norgate, 1878.

A. Ibn A. Z. AL-KAYRAWANI, La Risala..., trad. L. Bercher, Alger, J. Carbonel, 1952

Identités religieuses en Europe, dir. G. Davie et D. Hervieu-Léger, Paris, la Découverte, 1996.

A. D. EL-DJAZAÍRI, La voie du musulman, trad. M. Chakroun, Paris, Aslim éd., 1986.

Initiation à l'Islam, dir. M. Hamidullah, Paris, Amicale des musulmans de France, 1963.

\section{Ouvrages particuliers:}

$\mathrm{M}$. ABDESSELEM, Le thème de la mort dans la poésie arabe des origines à la fin du IIIe/IXe siècle, publication de l'Université de Tunis, 1977.

Z. BIANU, Les religions et... la mort, Paris, Ramsay, 1981

H. CORBIN, Terre céleste et corps de résurrection, Paris, Buchet-Chastel, 1960.

J. FAVRET-SAADA, Les mots, la mort, les sorts, Paris, Gallimard, 1977.

L. GARDET, L'Islam, religion et communauté, Paris, Desclée de Brouwer 1970.

F. HAJA, La mort et le Jugement Dernier selon les enseignements de l'Islam, s.l. (France), Arayhane éd., 1991.

F. HOVEYDA, L'Islam bloqué, Paris, R. Laffont, 1992.

F. KHOSROKHAVAR, L'islamisme et la mort. Le martyre révolutionnaire en Iran, Paris, L'Harmattan, 1995.

B. SADR, Le Coran et le pouvoir, Paris, Imago, 1993

L.-V. THOMAS, Rites de mort, Paris, Fayard, 1985.

E. WEBER, L'Islam sunnite traditionnel, [Turnhout], Brepols, 1993.

\section{Articles et revues:}

M. ABDESSELEM, article sur la mort dans Encyclopédie de l'islam, coll., tome VI, Brill, Maisonneuve, Leiden, Paris, 1986, p. 902.

S. E. BARIKI, "Identité religieuse, identité culturelle en situation immigrée ", dans Annuaire de l'Afrique du Nord, vol. XXIII, 1984, p. 427-445.

W. C. CHITTICK, "Your Sight Today is Piercing: the Muslim Understanding of Death and Afterlife ", dans Death and Afterlife. Perspectives of World Religions, éd. H. Obayashi, New York, London, Praeger, 1969, p. 125-139.

D. SOURDEL, "Le jugement des morts dans l'Islam ", dans Le jugement des morts, Sources orientales, vol. IV, Paris, Seuil, 1961, p. 179-204.

A. T. WELCH, "Death and dying in the Qur'an ", dans Religious Encounters with Death, éd. F.E. Reynolds and E.H. Wangh, Univ. Press, Pennsylvania, 1977, p. 183-199. 